\title{
Generalized Convulsions- a rare Presentation of HaNDL Syndrome
}

\author{
Geetanjali S Rathore* \\ Department of Pediatrics, University of Nebraska Medical Center, USA
}

*Corresponding author: Geetanjali S Rathore, Pediatric Neurology, Division Chief., Department of Pediatrics, University of Nebraska Medical Center, Department of Pediatrics, 982155 Nebraska Medical Center, Omaha, NE, USA.

To Cite This Article: Geetanjali S Rathore, Generalized Convulsions- a rare Presentation of HaNDL Syndrome. 2020 - 10(2). AJBSR.MS.ID.001484. DOI: $10.34297 /$ AJBSR.2020.10.001484.

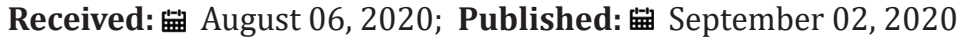

\begin{abstract}
Headache with Neurologic Deficits and cerebrospinal fluid Lymphocytosis (HaNDL) syndrome is a rare entity related to migrainous headaches. It is a benign, self-limited disorder, which is characterized by transient neurological symptoms and cerebrospinal fluid lymphocytosis. The International Headache Society criteria requires, episodes involve migraine-like headache along with weakness, sensory changes, and/or aphasia lasting at least 4 hours. The cerebrospinal fluid is notably abnormal, with an elevated protein and lymphocyte count greater than 15 cells $/ \mu \mathrm{L}$. Episodes typically recur but resolve without long-term sequalae. The most frequent neurologic signs are hemiparesis, hemisensory disturbances, and aphasia. Electroencephalogram (EEG) studies have shown that patients with HaNDL may have non-specific non-epileptiform changes such as asymmetrical generalized slowing, intermittent rhythmic delta activity and triphasic waves on their EEG. On our literature review, there are no reports of convulsive seizures that have been reported as a symptom of HaNDL. Our patient met the diagnostic criteria of HaNDL but had a unique neurological symptom of convulsive seizures.
\end{abstract}

Keywords: HaNDL Syndrome, Lymphocytosis, Migraine, Convulsion, Transient neurological deficit, Stroke mimics

Abbreviations: HaNDL: Headache and Neurologic Deficits with Cerebrospinal Fluid Lymphocytosis; EEG: Electroencephalogram; CT: Computerized tomography; CSF: Cerebrospinal Fluid; CRP: C-reactive Protein; ESR: Erythrocyte Sedimentation Rate; ANA: Antinuclear Antibodies

\section{Introduction}

The syndrome of transient headache and neurological deficits with cerebrospinal fluid lymphocytosis (HaNDL) is a rare disorder with a benign and self-limiting course [1]. This syndrome may mimic common conditions such as stroke. complex or hemiplegic migraine, aseptic meningitis, or meningoencephalitis. Ruling out these various serios illnesses through vigorous testing is required before this diagnosis of exclusion can be reached [2]. Seizures have not been reported as a presenting symptom of HaNDL syndrome, even though electroencephalogram (EEG) abnormalities are well documented in these patients [3]. Our patient met the criteria for diagnosis HaNDL but had a previously unreported presentation with generalized convulsive seizures.

\section{Material and Methods}

A 13-year-old healthy young girl presented with severe headache she developed during bowling. She suddenly fell to the ground after developing severe headache and was noted to have generalized stiffening and possible convulsive activity for a few seconds. She was noted to be febrile to 103-degree Fahrenheit in the emergency room. She underwent a computerized tomography (CT) of her head, which was normal. Lumbar puncture was done which showed cerebrospinal fluid (CSF) to have a white cell count of $29 / \mathrm{mm} 3$ with lymphocyte predominance, elevated protein at $75 \mathrm{mg} / 100 \mathrm{ml}$, normal glucose. Viral and bacterial panels on CSF were negative for any infectious source. She received hydration and non-steroidal analgesics which helped with resolution of her headaches in 48 hours. An electroencephalogram (EEG) done as outpatient was normal. The patient remained asymptomatic for several months before she presented to the hospital again with severe headache and fever. In the hospital patient had two episodes of couple minutes of generalized tonic -clonic seizure activity with postictal confusion. She was started on prophylactic anticonvulsant 
and empiric antibiotics including acyclovir, in addition to hydration and nonsteroidal for pain management. She underwent another lumbar puncture which showed a high CSF white count of $34 / \mathrm{mm} 3$ with elevated protein at $103 \mathrm{mg} / 100 \mathrm{ml}$. Magnetic resonance imaging (MRI) of the brain showed left hemispheric Dural enhancement and thickening, which can be concerning for meningitis. An extensive infectious workup was negative. Repeat EEG was nonspecific with mild intermittent slowing over left hemisphere. a comprehensive autoimmune work up including C-reactive protein (CRP), erythrocyte sedimentation rate (ESR), Antinuclear antibodies (ANA) panel, Autoimmune encephalopathy panel, were all negative. Her symptoms resolved within 48 hours. She has remained headache and seizure free for the last 2 months.

\section{Results and Discussion}

The syndrome of Transient Headache with Neurological Deficits and Lympocytosis (HaNDL) was first described by Swanson, Bartleson \& Whisnant [4]. It is a benign condition characterized by one or more episodes of severe headache, transient neurologic deficits, and CSF lymphocytic pleocytosis. As seen in our patient, the onset of headache is often preceded by symptoms suggestive of an infection and/or fever [5]. It is reported in both adults and children, but rare in children, most commonly presenting in third to fourth decade [6]. Majority of patients do not have a previous history of migraine headaches and unlike migraine, it has a slight predilection for males [5].

According to the International Classification of Headache Disorders, the diagnostic criteria for HaNDL syndrome include

1) episodes of moderate or severe headache lasting hours before resolving fully;

2) CSF pleocytosis with lymphocytic predominance (>15 cells/mm3)

3) episodes of headache are accompanied by or shortly follow transient neurologic deficits

4) episodes of headache and neurologic deficits recur over fewer than 3 months [7].

Our patient was a young female presenting with severe headache and convulsions, a rare presentation for HaNDL syndrome. Having had 2 episodes of severe headache within couple of months, preceded by fever, spinal fluid showed lymphocytosis, she met the first two requirements of the diagnostic criteria for HaNDL syndrome. Her focal neurological symptoms of convulsive seizures however were extremely atypical and never been reported in the literature. There is clear evidence of EEG abnormalities reported in patients with HaNDL syndrome, most commonly presenting as focal or generalized delta/theta slowing of the background [8]. Multifocal and other epileptiform discharges have also been reported, but a clinical seizure has never been described with HaNDL syndrome [9].

As this condition can mimic extremely serious conditions like meningitis, stroke, aneurysm, structural brain lesion and intracranial bleeding, it is a diagnosis of exclusive. A thorough workup was done in our patient to rule these out. Her extensive infectious workup in blood and CSF remained negative during both episodes. She did not have any evidence of stroke, brain lesion, intracranial bleeding, or aneurysm. Inflammatory markers, autoimmune antibodies, rheumatological panels were all unremarkable. Her brain imaging did show dural enhancement and thickening over the left hemisphere. Magnetic resonance imaging (MRI) of the brain is typically normal in most patients with HaNDL syndrome. There are however multiple reports describing abnormalities on brain imaging. Most commonly evidence of impaired perfusion is noted on CT perfusion scans, Single photon emission computed tomography (SPECT) and Transcranial doppler (TCD) show unilateral or focal hypoperfusion [10]. MRI is not helpful in detecting perfusion abnormalities but has been reported to show meningeal enhancement in the temporal and occipital hemispheres [11], as seen in our patient.

The underlying pathophysiology of HaNDL syndrome is unclear, however postulated to be migrainous or an infectious/postinfectious process [12]. The findings of focal impaired neuronal metabolism and hypoperfusion suggest a migrainous mechanism $[10,13]$. However, the abnormal CSF, predilection for males, no history of migraine headaches, makes the atypical migraine hypothesis less likely. The presence of fever and CSF lymphocytosis indicates possibility of meningoencephalitis as a possible mechanism. This theory is contraindicated by the negative infectious workup in all patients except less than a handful of reports suggesting viral presence $[14,15]$. Cortical irritation from an aseptic meningitis that triggers a migraine-like cortical spreading depression appears to be the most plausible etiology that explains the presentation of HaNDL syndrome $[5,16]$.

Treatment is symptomatic and supportive with analgesics and antiemetics [1,17]. These episodes can mimic serious conditions and cause significant stress, education and reassurance of the patient and family are of equal importance.

\section{Conclusion}

HaNDL syndrome is a rare condition in children presenting with self-limiting headache and neurological deficits in the presence of CSF lymphocystosis. These episodes may mimic serious conditions like stroke, meningitis, aneurysm or intracranial lesion and HaNDL is diagnosed after excluding these etiologies.The most common 
neurological symptoms comprise of hemiparesis, hemisensory changes, aphasia and confusion. As seen in our patient, a convulsive seizure can also be a presenting symptom. HaNDL syndrome should be considered if all other investigations are unrevealing for an epileptogenic etiology.

\section{Acknowledgement}

A verbal consent was obtained from the patient's family with the patient's assent.

\section{Conflict of Interest}

The authors have no conflict of interest to report.

\section{References}

1. Gómez-Aranda, F, Cañadillas, F, Martí-Massó, R Leira, M Gracia, et al. (1997) Pseudomigraine with temporary neurological symptoms and lymphocytic pleocytosis. A report of 50 cases. Brain 120: 1105-1113.

2. Rivero-Sanz E, Pias-Peleteiro L, Gonzalez-Alvarez (2016) HaNDL syndrome in a 14-year-old girl. BMJ Case Rep bcr2015213018.

3. Berg, MJ, Williams, LS (1995) The transient syndrome of headache with neurologic deficits and CSF lymphocytosis. Neurology 45: 1648-1654

4. Bartleson, JD, Swanson, JW, Whisnant, JP (1981) A migrainous syndrome with cerebrospinal fluid pleocytosis. Neurology 31:1257-1262.

5. A Cifelli, L Vaithianathar (2011) Syndrome of transient Headache and Neurological Deficits with cerebrospinal fluid Lymphocytosis (HaNDL). BMJ Case Rep bcr0320102862.

6. Rossi LN, Vassella F, Bajc O (1985) Benign migraine-like syndrome with CSF pleocytosis in children. Dev Med Child Neurol 27: 192-198.

7. (2013) Headache Classification Committee of the International Headache Society. The International Classification of Headache Disorders, 3rd Edition (beta). Cephalalgia 33(9): 719.
8. Parissis D, Ioannidis P, Balamoutsos G (2011) Confusional state in the syndrome of HaNDL. Headache 51: 1285-1288.

9. Elena Rivero-Sanz, Leticia Pias-Peleteiro, Veronica Gonzalez-Alvarez (2016) HaNDL syndrome in a 14-year-old girl. BMJ Case Rep bcr2015213018.

10. Kappler, J, Mohr, S, Steinmetz, H (1997) Cerebral vasomotor changes in the transient syndrome of headache with neurologic deficits and CSF lymphocytosis (HaNDL). Headache 37: 516-518.

11. Yilmaz, A, Kaleagasi, H, Dogu, E Kara, A Ozge (2010) Abnormal MRI in a patient with 'headache with neurological deficits and CSF lymphocytosis (HaNDL). Cephalalgia 30(5): 615-619.

12. Pascual J, Valle N (2003) Pseudomigraine with lymphocytic pleocytosis. Curr Pain Headache Rep 7(3): 224-248.

13. Schraeder PL, Burns RA (1980) Hemiplegic migraine associated with an aseptic meningeal reaction. Arch Neurol 37(6): 377-379.

14. Vicente Mas J, Juni Sanahuja J, Benítez Bermejo R, et al. (2004) Cytomegalovirus infection mimicking a pseudomigraine with pleocytosis. A Med Interna 21: 360-361.

15. Castels-van Daele M, Standaert L, Boel M, et al. (1981) Basilar migraine and viral meningitis. Lancet 1: 1366.

16. Bolay H, Reuter U, Dunn AK, Zhihong Huang, David A Boas, et al. (2002) Intrinsic brain activity triggers trigeminal meningeal afferents in a migraine model. Nat Med 8(2): 136-142.

17. Moavero, R, Papetti, L, Tarantino S, Barbara Battan, Irene Salfa, et al. (2018) Syndrome of transient headache and neurologic deficits with cerebrospinal fluid lymphocytosis should be considered in children presenting with acute confusional state. Headache 58(3): 438-442. 